\title{
The 2000 Rosetta asteroid targets observational campaign: 140 Siwa and 4979 Otawara
}

\author{
A. Le Bras ${ }^{1,4}$, E. Dotto $^{3}$, M. Fulchignoni ${ }^{1,2}$, A. Doressoundiram ${ }^{1}$, M. A. Barucci ${ }^{1}$, S. Le Mouélic ${ }^{4}$, \\ O. Forni ${ }^{4}$, and E. Quirico ${ }^{4}$ \\ 1 DESPA, Observatoire de Paris, 5 place Jules Janssen, 92195 Meudon, France \\ 2 UFR de Physique Université Denis Diderot - Paris 7, 2 place Jussieu 75005 Paris, France \\ 3 Osservatorio Astronomico di Torino, 10025 Pino Torinese, Italy \\ 4 Institut d'Astrophysique Spatiale, Bât. 121, Université Paris-Sud, 91405 Orsay, France
}

Received 11 July 2001 / Accepted 18 September 2001

\begin{abstract}
Photometric and spectroscopic observations of 140 Siwa and 4979 Otawara, targets of the Rosetta mission, have been carried out at the European Southern Observatory (ESO, Chile). The complete lightcurves in $V$ and $R$ of both asteroids and the Siwa spectrum in the range $0.95-2.52 \mu \mathrm{m}$ have been obtained. We determined the precise synodic rotational period of 140 Siwa $(18.495 \mathrm{~h} \pm 0.005 \mathrm{~h})$ and we confirmed the synodic rotational period of 4979 Otawara $(2.707 \mathrm{~h} \pm 0.005 \mathrm{~h})$. The phase function has been obtained for both asteroids, allowing the determination of the $H_{V}$ and $G_{V}$ parameters: for 4979 Otawara $H_{V}=14.62 \mathrm{mag}, G_{V}=0.34$; for 140 Siwa $H_{V}=8.34 \mathrm{mag}, G_{V}=0.17$. The near infrared spectrum of Siwa does not show any spectral feature, which is consistent with a $\mathrm{C} / \mathrm{P}$ type object.
\end{abstract}

Key words. minor planets, asteroids - solar system: general

\section{Introduction}

Asteroids 4979 Otawara and 140 Siwa are the targets of the 2006 and 2008 fly-bys of the European Space Agency cornerstone Rosetta mission on its way to the rendezvous with the comet Wirtanen in 2011. The scientific goals of these fly-bys are: i) the characterization of global physical properties of the asteroids: size, shape, pole direction, rotational period, density and mass; ii) the study of the morphological and compositional characteristics; iii) the exploration of the asteroids environment, to detect the presence of dust and/or gas and potential satellites.

An international observational campaign has been organized since 1996 (Barucci et al. 1998; Doressoundiram et al. 1999; Doressoundiram et al. 2001) to characterize the physical and compositional properties of these asteroids in order to optimize both the mission trajectory and the science operations.

The 2000 observational campaign was particularly favorable. Both asteroids were at the opposition at the same time (August the 7th for 4979 Otawara and August the 9th for 140 Siwa) and within few degrees in the southern sky.

Send offprint requests to: A. Le Bras, e-mail: aurelie.lebras@obspm.fr
The observations were carried out at European Southern Observatory both for photometry (eight nights at the $1.5 \mathrm{~m}$ Danish telescope) and for spectroscopy (two nights at the 3.6 NTT telescope).

\section{Observations and data reduction}

\subsection{Photometry}

We observed 4979 Otawara and 140 Siwa during eight nights, in July (23, 24 and 28) 2000 and August (3,6-9) 2000. The purpose of these photometric observations was to obtain: i) the precise rotational period of Siwa; ii) lightcurves at different aspects for Siwa and Otawara; iii) phase curves of Siwa and Otawara. We observed with the $1.5 \mathrm{~m}$ Danish telescope at the European Southern Observatory on La Silla (Chile). The telescope is equiped with a Loral/Lesser CCD detector $(2052 \times 2052$ pixels $)$, mounted on DFOSC (Danish Faint Object Spectrograph and Camera), a focal reducer instrument. The field is $13.3^{\prime} \times 13.3^{\prime}$ and the image scale is 0.39 arcsec per pixel. We used Bessel $U B V R$ and Gunn $I$ broad band filters. The nights of August 6-8 were photometric, with an average seeing of 1-1.2 arcsec. The nights of August 3 and 9 were not photometric, with an average seeing around 2 arcsecs.

The images were reduced in a standard manner: average bias substraction, division by a mean skyflat. 
Table 1. Observational characteristics of 140 Siwa and 4979 Otawara. Values of $r, \Delta$ and $\alpha$ are given at 0 h00 UT for photometry, and at the time of the beginning of the spectrum acquisition for spectroscopy.

\begin{tabular}{lccc}
\hline Date & $r$ & $\Delta$ & $\alpha$ \\
& $(\mathrm{AU})$ & $(\mathrm{AU})$ & $(\mathrm{deg})$ \\
\hline 140 Siwa: photometry \\
24 Jul. 2000 & 2.1460 & 1.1596 & 8.844 \\
25 Jul. 2000 & 2.1463 & 1.1567 & 8.839 \\
29 Jul. 2000 & 2.1478 & 1.1471 & 6.287 \\
04 Aug. 2000 & 2.1505 & 1.1397 & 3.217 \\
07 Aug. 2000 & 2.1520 & 1.1391 & 1.883 \\
08 Aug. 2000 & 2.1525 & 1.1394 & 1.572 \\
09 Aug. 2000 & 2.1531 & 1.1400 & 1.413 \\
10 Aug. 2000 & 2.1536 & 1.1407 & 1.457 \\
\hline 4979 Otawara: photometry \\
24 Jul. 2000 & 1.8921 & 0.8953 & 8.634 \\
25 Jul. 2000 & 1.8930 & 0.8936 & 8.022 \\
29 Jul. 2000 & 1.8968 & 0.8892 & 5.541 \\
04 Aug. 2000 & 1.9028 & 0.8891 & 1.879 \\
07 Aug. 2000 & 1.9060 & 0.8921 & 0.982 \\
08 Aug. 2000 & 1.9070 & 0.8935 & 1.322 \\
09 Aug. 2000 & 1.9081 & 0.8952 & 1.827 \\
10 Aug. 2000 & 1.9092 & 0.8970 & 2.394 \\
\hline \hline 140 Siwa: spectroscopy \\
18 Aug. 2000 & 2.1587 & 1.1559 & 4,989 \\
20 Aug. 2000 & 2.1600 & 1.1621 & 6.028 \\
\hline
\end{tabular}

Calibration was performed with standard stars (Landolt 1992), when nights were photometric. Data acquired in August were reduced as a whole with the ESO software MIDAS. Bias of each night was a mean of bias at the beginning and the end of the night. Photometric flat fields were obtained during twilight, before and after the night. For the night of 3 August, the mean flat field is the mean of all the flats of the night, as well as for the 6. A superflat, mean of all the flats of the 7, 8 and 9 August was calculated. Absolute calibration of the nights of 6,7 and 8 August was performed with SA 92 335, Mark A and PG1525-071 (Landolt 1992). The field of Otawara on the night of 3 August was observed during the night of 8 August, in order to calibrate in absolute magnitude this field (night of 3 August was not photometric). Instrumental magnitudes were calculated with the procedure magnitude/circle: the flux of the inner circle corresponding to the source minus the flux of the sky.

\subsection{Spectroscopy}

We observed 140 Siwa during the ends of two nights using the SOFI instrument mounted on the $3.6 \mathrm{~m}$ New Technology Telescope (NTT) at La Silla Observatory (Chile). The spectral domain is 0.95 to $2.52 \mu \mathrm{m}$, divided into two parts. A first grism covers the $0.95-1.64 \mu \mathrm{m}$ range while a second grism covers the $1.53-2.52 \mu \mathrm{m}$ range. This spectral domain allows the detection of the main absorption features of mafic silicates such as bands at 1 and $2 \mu \mathrm{m}$ when present. We used a slit size of $2^{\prime \prime}$ which gives a spectral resolution of $\sim 300$.

The blue part of the spectrum was acquired at 4h 30 UT the 08-20-2000, with an airmass ranging from 1.7 to 1.9 during the data acquisition run. The red part of the spectrum was acquired at $3 \mathrm{~h} 30 \mathrm{UT}$ the $08-18-2000$ with an airmass ranging from 1.36 to 1.73 . Corrections for the proper motion of the asteroid have been introduced to keep the target in the center of the slit aperture. The sky background was measured and removed from the observations. Observations of a G2V "solarlike" nearby star (HD 190617) were performed before and after Siwa observations. This calibration star provides both atmospheric and instrumental calibration. As a test of our reduction process, we have also observed the asteroid 4 Vesta, which spectral properties in the $0.9-2.5 \mu \mathrm{m}$ domain are already well known (Gaffey 1983). A good consistency was obtained with previous observations (Le Mouélic et al. 2001).

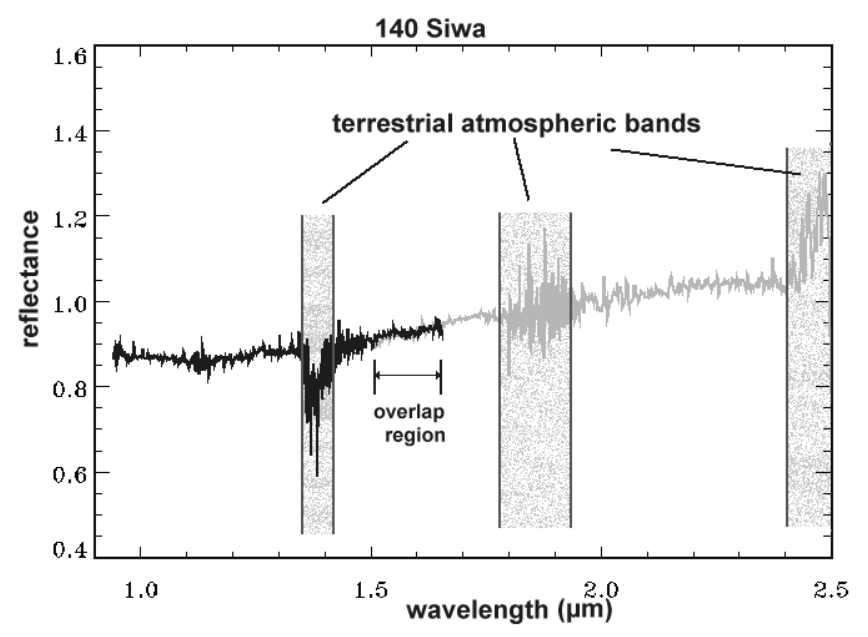

Fig. 1. Normalized infrared composite spectrum of 140 Siwa. Residual terrestrial atmospheric features at $1.4 \mu \mathrm{m}, 1.9 \mu \mathrm{m}$ and $2.5 \mu \mathrm{m}$ should not be considered. No deep absorption feature corresponding to mafic minerals appears in this spectrum, which is consistent with 140 Siwa belonging to the C class.

\section{140 Siwa}

140 Siwa is a main belt asteroid with a diameter of $110 \mathrm{~km}$ and an IRAS albedo of 0.068 (Tedesco et al. 1992). Tholen et al. (1979) classified Siwa as a P type, Barucci et al. (1998) assigned the asteroid to the C class while Bus (1999) obtained a classification in his system of $X_{\mathrm{c}}$. All these classes correspond to a flat or a slightly reddish spectrum. No evidence of aqueous alteration nor morphological variations with the rotation on its surface have been found. An estimation of the rotational period larger than 22 hours was given by Schober et al. (1979) and Harris et al. (1980), while Lagerkvist et al. (1979) gave a value of 18.5 hours. On the basis of our observations (see Table 1) we determined the asteroid synodic rotational period and the corresponding uncertainty of 18.495 hours \pm 0.005 hours 

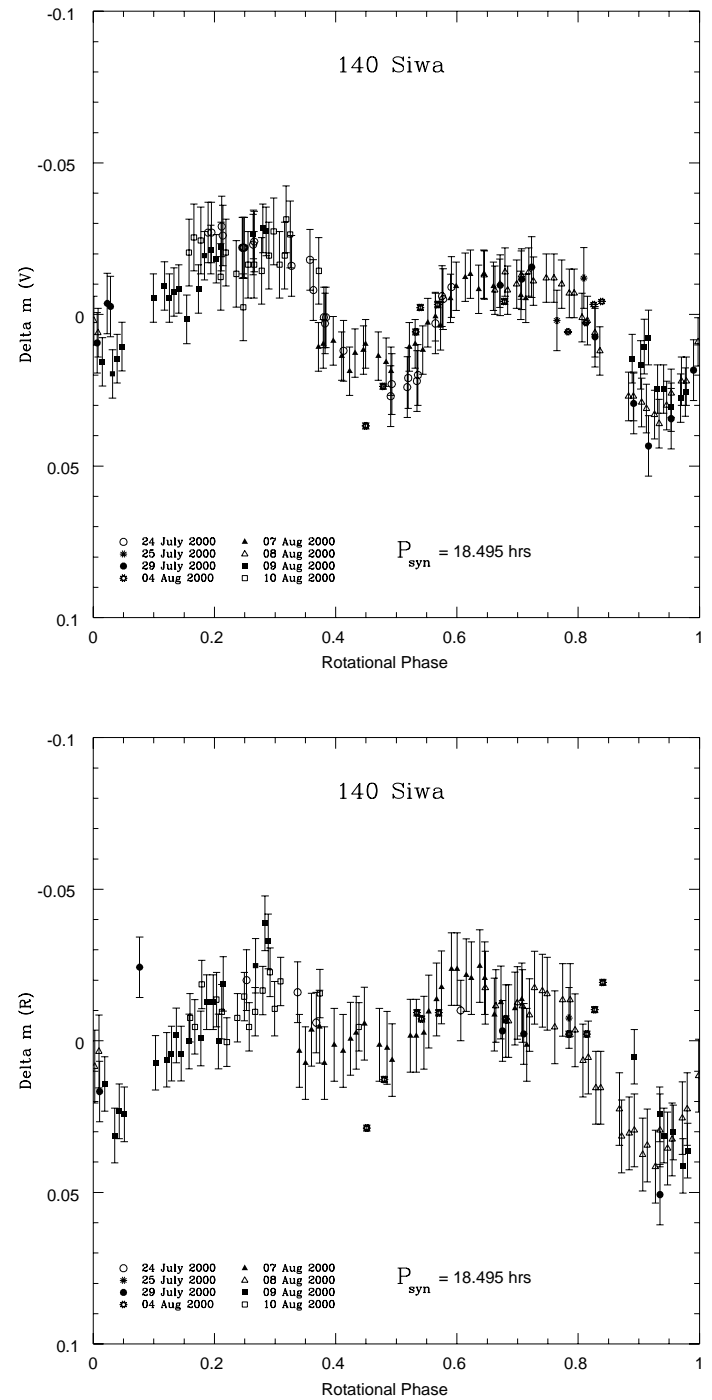

Fig. 2. Composite lightcurves of 140 Siwa, in $V$ (up) and in $R$ (down). The zero phase is at JD 2451761.0.

(Fig. 2) by applying the Fourier analysis as described in Harris et al. (1980). The composite $V$ and $R$ lightcurves have the same amplitude of $0.05 \pm 0.01 \mathrm{mag}$, and they have also the same shape: they show two maxima and two slightly asymmetric minima.

The phase function (absolute magnitude vs. phase angle) has been obtained using the mean magnitude of each $V$ lightcurve (Fig. 3). We determined the $H_{V}$ and $G_{V}$ parameters defining the IAU magnitude system for asteroids (Bowell et al. 1989):

$$
H_{V}=8.34 \pm 0.04 \mathrm{mag} \quad G_{V}=0.17 \pm 0.01
$$

The near infrared spectrum of Siwa is shown in Fig. 1. There is a good consistency between the spectrum acquired with the first grism $(0.95-1.64 \mu \mathrm{m})$ and the spectrum acquired with the second grism $(1.53-2.52 \mu \mathrm{m})$ in the overlap region. The spectrum does not show any spectral feature, which confirms the $\mathrm{C} / \mathrm{P}$ type nature of Siwa. Taking into account the rotational period, the two parts

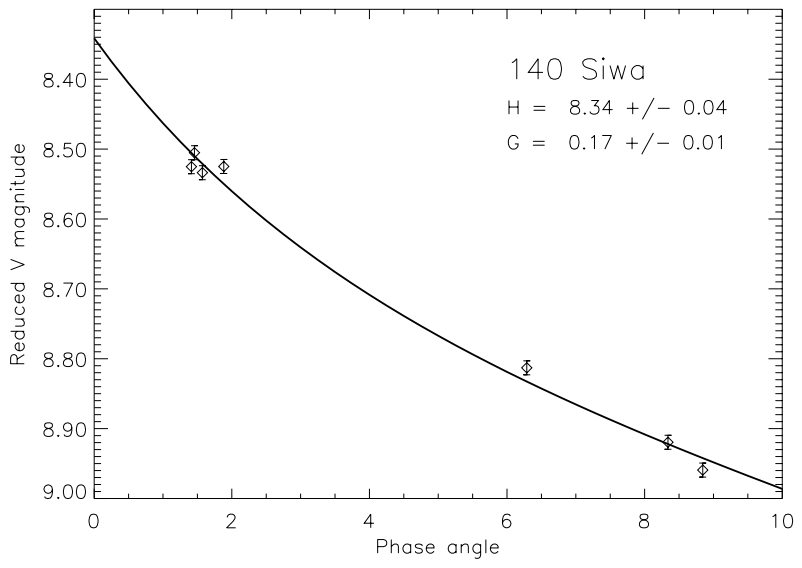

Fig. 3. Phase function of 140 Siwa obtained in $V$.

of the spectrum can refer to two different hemispheres of the asteroid. The flatness of both parts indicates that the spectral characteristics of the two hemispheres are very similar, at least with this kind of observation with no spatial resolution.

\section{4979 Otawara}

4979 Otawara is a small asteroid with a diameter of the order of 5 kilometers. An almost complete overview of the asteroid rotational and compositional properties is given in Doressoundiram et al. (1999).



Fig. 4. Phase function of 4979 Otawara obtained in $V$.

On the basis of our observations, we confirmed the value of $2.707 \pm 0.005$ hours for the synodic rotational period of Otawara. The obtained composite $V$ lightcurve (Fig. 5) has an amplitude of $0.22 \pm 0.01 \mathrm{mag}$ (respectively $0.22 \pm 0.02 \mathrm{mag}$ in $R$ ). The $V$ and $R$ lightcurves have the same double peak shape and present the same behavior of the lightcurves obtained during the 1998/99 opposition.

The phase function (Fig. 4) allowed us to determine the following $H_{V}$ and $G_{V}$ parameters:

$H_{V}=14.62 \pm 0.04 \mathrm{mag} \quad G_{V}=0.34 \pm 0.03$ 

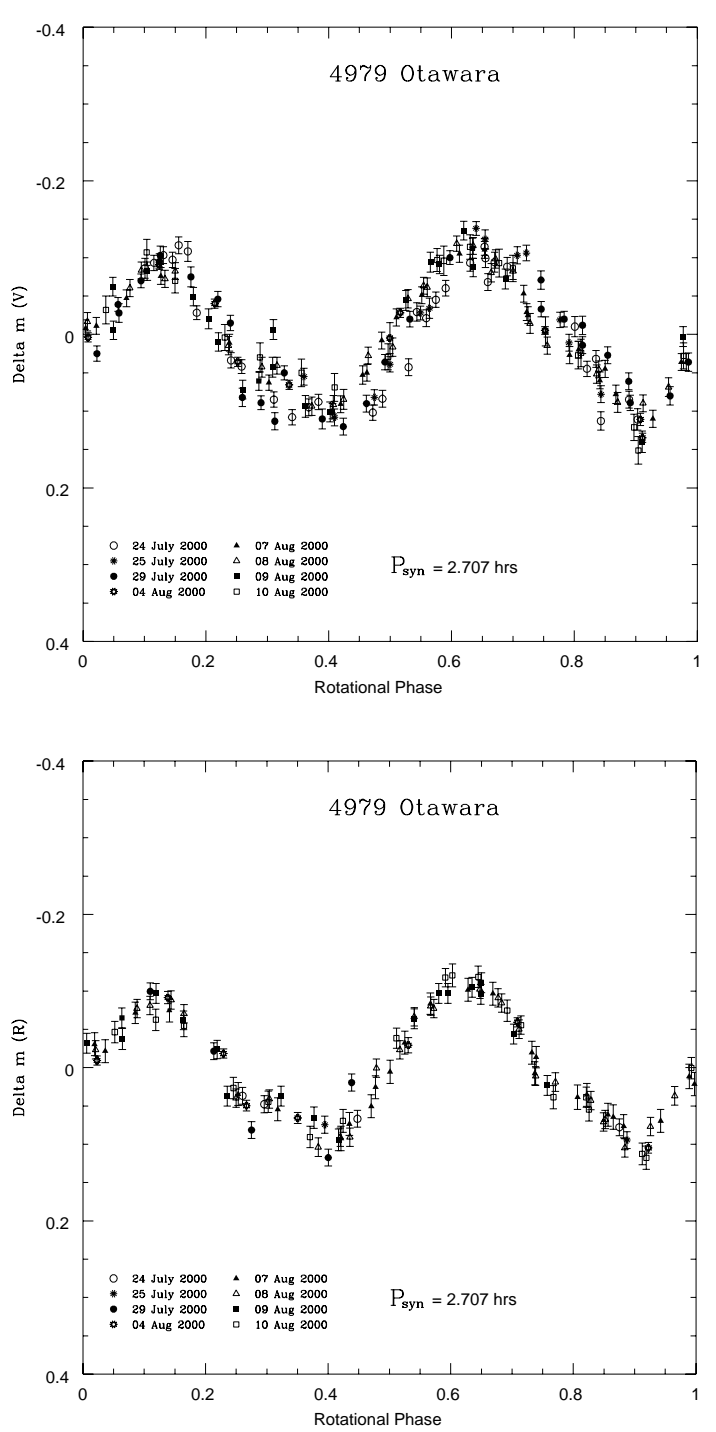

Fig. 5. Composite lightcurves of 4979 Otawara, in $V$ (up) and in $R$ (down). The zero phase is at JD 2451759.0.

\section{Conclusions}

During the 2000 oppositions of the asteroids 140 Siwa and 4979 Otawara we obtained:

1. the precise determination of the synodic rotational period of Siwa and we confirmed the previous determination of that of Otawara.

2. the phase functions for both asteroids which allowed us to determine the $\mathrm{H}$ and $\mathrm{G}$ parameters of the IAU magnitude system for asteroids.

3. the near IR spectrum of Siwa.

The length of the rotational period of 140 Siwa will not allow the Rosetta remote sensing instruments to cover the whole asteroid surface at high resolution, even if its size will allow an early start of the disc-resolved observations. The fast rotation of 4979 Otawara will allow the on-board Rosetta instruments to measure the asteroid surface characteristics during one complete rotation of the asteroid at high resolution. The $G$ values obtained for both asteroids are in good agreement with the average values estimated for the asteroids of the same types $(S / V$ for 4979 Otawara and $\mathrm{C} / \mathrm{P}$ for 140 Siwa). The $H_{V}$ value obtained for Siwa coincides with the value used in the IRAS Minor Planet (Tedesco et al. 1992). Further CCD photometric observations near opposition, occurring at different celestial latitudes, are necessary in order to determine the pole direction of both target asteroids. Additional spectroscopic observations of 4979 Otawara are needed i) in the near IR, to state definitely its spectral type ( $S$ or $V$ ) and ii) in visible and near infrared wavelengths along a complete rotation, to detect possible variations (morphological/compositional) of the asteroid surface. The knowledge of all these parameters is needed in order to optimize the scientific return of the fly-by of the Rosetta target asteroids.

Acknowledgements. We thank A. Erikson for his kind collaboration.

\section{References}

Bowell, E., Hapke, B., Domingue, D., et al. 1989, in Asteroid II, ed. R. P. Binzel, T. Geherels, \& M. Shapley Matthews (The University of Arizona Press, Tucson), 524

Barucci, M. A., Doressoundiram, A., Fulchignoni, M., et al. 1998, P\&SS 46, 75

Bus, S. J., Compositional structure in the asteroid belt: Results of a spectroscopic survey, Ph.D. Thesis, Massachusetts Institute of Technology

Doressoundiram, A., Fulchignoni, M., \& Barucci, M. A. 2001, ESA SP-1165, in press

Doressoundiram, A., Weissman, P. R., Fulchignoni, M., et al. 1999, A\&A, 352, 697

Gaffey, M. J. 1983, LPSC XIV, 231

Harris, A. W., \& Young, J. W. 1980, Icarus 43, 20 Harris, A. W., Young, J. W., Bowell, E., et al. 1989, Icarus 77,171

Lagerkvist, C.-I., Magnusson, P., Debehogne, H., et al. 1992, A\&ASS, 95, 461

Landolt, A. U. 1992, ApJ, 104, 340

Le Mouélic, Forni, O., \& Quirico, E. 2001, LPSC XXXII, 1094

Schober, H. J., \& Stanzel, R. 1979, A\&AS, 38, 265

Tedesco, E. F., Veeder, G. J., Fowler, G. W., et al. 1992, IRAS minor planets survey, PL-TR-922049

Tholen, D. J. 1989, in Asteroid II, ed. R. P. Binzel, T. Geherels, \& M. Shapley Matthews (The University of Arizona Press, Tucson), 1139 